\title{
Genetic testing for large-caliber vessel aneurysms
}

\author{
Yeltay Rakhmanov ${ }^{1}$, Paolo Enrico Maltese ${ }^{1 \star}$, Alessandra Zulian ${ }^{2}$ and Matteo Bertelli1 ${ }^{1,2}$
}

\begin{abstract}
Large-caliber vessels are those with a diameter of $10 \mathrm{~mm}$ or more. Most aneurysms remain asymptomatic until they expand or rupture. Aortic aneurysms are of special interest for physicians and scientists because of their prevalence. Aortic aneurysms and dissections account for 1-2\% of all deaths in western countries. Expansion and rupture of vascular aneurysms show a strong correlation with hyperlipidemia, hypertension, smoking, sex and age. Heritability estimates have been as high as $70 \%$. This Utility Gene Test was developed on the basis of an analysis of the literature and existing diagnostic protocols. It is useful for confirming diagnosis, as well as for differential diagnosis, couple risk assessment and access to clinical trials.
\end{abstract}

Keywords: Large-caliber vessel aneurysms, EBTNA UTILITY GENE TEST

'MAGI's Lab, Rovereto, Italy

${ }^{2}$ MAGI Euregio, Bolzano, Italy

*Corresponding author: P. E. Maltese E-mail: paolo.maltese@assomagi.org

DOI: 10.2478/ebtj-2018-0044
(C) 2018 Authors. This work was licensed under the Creative Commons AttributionNonCommercial-NoDerivs 3.0 License.

\section{General information about the disease}

Large-caliber vessels have a diameter of at least $10 \mathrm{~mm}$. Aneurysms in the following vessels are included in this group: ascending aorta, aortic arch, descending thoracic aorta (subdiaphragmatic aorta, infra-diaphragmatic aorta), suprarenal abdominal aorta, infra-renal aorta, brachycephalic artery, subclavian artery, common carotid artery, common iliac artery, axillary vein, retro-aortic innominate vein, superior vena cava, common femoral vein, external iliac vein, common iliac vein, inferior vena cava, pulmonary arteries and pulmonary veins. Due to their clinical importance, the etiology and genetic causes of aneurysms of large-caliber arteries have been well studied, whereas there is little literature on vein aneurysms due to an absence of specific symptoms, unless they are complicated by their various manifestations, one being deep vein thrombosis (1).

More than $80 \%$ of cases of pulmonary venous aneurysm are congenital and half or more are associated with hereditary hemorrhagic telangiectasia (2). Pulmonary artery dilation is a minor cardiovascular criterion in the diagnosis of Marfan syndrome (3).

Aortic aneurysms include thoracic aortic aneurysm (AAT) and abdominal aortic aneurysm (AAA) and account for 1-2\% of all deaths in western countries. Heritability estimates have been as high as 70\% (4-9).

The incidence of AAT is $\approx 10.4$ per 100000 person-years.

Abdominal aortic aneurysms are strongly linked to hyperlipidemia, hypertension, smoking, sex and age. The incidence of AAAs is about $8 \%$ among men over the age of 60 years (10).

Little is known about the pathogenesis of aortic aneurysms, however chronic inflammatory processes have a significant effect on the development of this defect. Upregulation of cytokine pathways associated with increased matrix turnover by matrix metalloproteinases (MMPs) has been observed $(10,11)$. In this regard, genetic influences on the regulation of inflammatory responses plays crucial role (12).

Prevalence is unknown.

Vascular aneurysms remain undiagnosed until they expand or rupture, when they 
can give rise to local compression, including early satiety, nausea, vomiting, urinary symptoms, vein thrombosis due to venous compression, abdominal pain, groin pain and embolic phenomena affecting the toes (13).

Diagnosis is based on routine physical examination, palpation, arterial blood pressure, chest radiogram, pulse oximetry, Doppler and two-dimensional echocardiography, spin-echo MR imaging, CT and genetic testing.

The condition can be part of an inherited syndrome or can manifest as an isolated trait, whether sporadic or affecting more than one family member.

Autosomal dominant non-syndromic large-caliber vessel aneurysms

- Thoracic aortic aneurysm 4 (AAT4, OMIM disease 132900) - MYH11 (OMIM gene 160745);

- Thoracic aortic aneurysm 6 (AAT6, OMIM disease 611788) - ACTA2 (OMIM gene 102620);

- Thoracic aortic aneurysm 7 (AAT7, OMIM disease 613780) - MYLK (OMIM gene 600922);

- Thoracic aortic aneurysm 8 (AAT8, OMIM disease 615436) - PRKG1 (OMIM gene 176894);

- Thoracic aortic aneurysm 9 (AAT9, OMIM disease 616166) - MFAP5 (OMIM gene 601103);

- Thoracic aortic aneurysm 10 (AAT10, OMIM disease 617168) - LOX (OMIM gene 153455);

- Thoracic aortic aneurysm 11 (AAT11, OMIM disease 617349) - FOXE3 (OMIM gene 601094);

- Aortic valve disease 1 (AOVD1, OMIM disease109730) NOTCH1 (OMIM gene 190198).

Autosomal dominant syndromic large-caliber vessel aneurysms

- Ehlers-Danlos syndrome, vascular type (EDSVASC, OMIM disease 130050) - COL3A1 (OMIM gene 120180);

- Loeys-Dietz syndrome 1 (LDS1, OMIM disease 609192) TGFBR1 (OMIM gene 190181);

- Loeys-Dietz syndrome 2 (LDS2, OMIM disease 610168) TGFBR2 (OMIM gene 190182);

- Loeys-Dietz syndrome 3 (LDS3, OMIM disease 613795) SMAD3 (OMIM gene 603109);

- Loeys-Dietz syndrome 4 (LDS4, OMIM disease 614816) TGFB2 (OMIM gene 190220);

- Loeys-Dietz syndrome 5 (LDS5, OMIM disease 615582) TGFB3 (OMIM gene 190230);

- Marfan Syndrome (MFS, OMIM disease 154700) - FBN1 (OMIM gene 134797);

- Contractural arachnodactyly, congenital (CCA, OMIM disease 121050) - FBN2 (OMIM gene 612570);

- Cutis laxa, autosomal dominant (OMIM disease 123700) ELN (OMIM gene 130160);

- Shprintzen-Goldberg craniosynostosis syndrome with aortic aneurysm (SGS, OMIM disease 182212) - SKI (OMIM gene 164780) (14).
Autosomal recessive syndromiclarge-caliber vessel aneurysms

- Cutis laxa, autosomal recessive, type IB (ARCL1B, OMIM disease 614437) - EFEMP2 (OMIM gene 604633);

- Arterial tortuosity syndrome (ATS, OMIM disease 208050) SLC2A10 (OMIM gene 606145).

\section{$\mathrm{X}$-linked syndromic large-caliber vessel aneurysms}

- Meester-Loeys syndrome (MRLS, OMIM disease 300989) BGN (OMIM gene 301870);

- Heterotopia, periventricular (OMIM disease 300049) - FLNA (OMIM gene 300017).

Likely candidate genes for non-syndromic and syndromic large vessel aneurysm are: MAT2A (15), SMAD2 (16), COL5A1 and COL5A2 (17).

Pathogenic variants may include missense, nonsense, splicing, small insertions, deletions, indels and rarely gross deletions and/ or duplications.

\section{Aims of the test}

- To determine the gene defect responsible for the disease;

- To confirm clinical diagnosis;

- To assess the recurrence risk and perform genetic counselling for at-risk/affected individuals.

\section{Test characteristics}

\section{Specialist centers/ Published Guidelines}

Guidelines for clinical use of the test are described in Genetics Home Reference (ghr.nlm.nih.gov) and Gene Reviews (18).

\section{Test strategy}

Clinically distinguishable syndromes can be analyzed by sequencing only those genes known to be associated with that specific disease using Sanger or Next Generation Sequencing (NGS); if the results are negative, or more generally if clinical signs are ambiguous for diagnosis, a multi-gene NGS panel is used to detect nucleotide variations in coding exons and flanking introns of the above genes.

Potentially causative variants and regions with low coverage are Sanger-sequenced. Sanger sequencing is also used for family segregation studies.

Multiplex Ligation Probe Amplification (MLPA) is used to detect duplications and deletions in COL3A1, FBN1, SLC2A10 and FLNA.

To perform molecular diagnosis, a single sample of biological material is normally sufficient. This may be $1 \mathrm{ml}$ peripheral blood in a sterile tube with $0.5 \mathrm{ml} \mathrm{K}$ EDTA or $1 \mathrm{ml}$ saliva in a sterile tube with $0.5 \mathrm{ml}$ ethanol $95 \%$. Sampling rarely has to be repeated.

Gene-disease associations and the interpretation of genetic variants are rapidly developing fields. It is therefore possible that the genes mentioned in this note may change as new scientific data is acquired. It is also possible that genetic variants today defined as of "unknown or uncertain significance" may acquire clinical importance. 


\section{Genetic test results \\ Positive}

Identification of pathogenic variants in the above genes confirms the clinical diagnosis and is an indication for family studies.

A pathogenic variant is known to be causative for a given genetic disorder based on previous reports, or predicted to be causative based on loss of protein function or expected significant damage to proteins or protein/protein interactions. In this way it is possible to obtain a molecular diagnosis in new/other subjects, establish the risk of recurrence in family members and plan preventive and/or therapeutic measures.

\section{Inconclusive}

Detection of a variant of unknown or uncertain significance (VUS): a new variation without any evident pathogenic significance or a known variation with insufficient evidence (or with conflicting evidence) to indicate it is likely benign or likely pathogenic for a given genetic disorder. In these cases, it is advisable to extend testing to the patient's relatives to assess variant segregation and clarify its contribution. In some cases, it could be necessary to perform further examinations/tests or to do a clinical reassessment of pathological signs.

\section{Negative}

The absence of variations in the genomic regions investigated does not exclude a clinical diagnosis but suggests the possibility of:

- alterations that cannot be identified by sequencing, such as large rearrangements that cause loss (deletion) or gain (duplication) of extended gene fragments;

- sequence variations in gene regions not investigated by this test, such as regulatory regions (5' and 3' UTR) and deep intronic regions;

- variations in other genes not investigated by the present test.

\section{Unexpected}

Unexpected results may emerge from the test, for example information regarding consanguinity, absence of family correlation or other genetically-based diseases.

\section{Risk for progeny}

If the identified pathogenic variant has autosomal dominant transmission, the probability that an affected carrier transmit the disease variant to his/her children is $50 \%$ in any pregnancy, irrespective of the sex of the child conceived.

In autosomal recessive mutations, both parents are usually healthy carriers. In this case, the probability of transmitting the disorder to the offspring is $25 \%$ in any pregnancy of the couple, irrespective of the sex of the child. An affected individual generates healthy carrier sons and daughters in all cases, except in pregnancies with a healthy carrier partner. In these cases, the risk of an affected son or daughter is $50 \%$.

In X-linked transmission, affected males transmit the pathogenic variant to their daughters and the probability that a fe- male carrier transmit the pathogenic variant to her offspring is $50 \%$ in any pregnancy irrespective of the sex of the child conceived. Females who inherit the pathogenic variant are carriers and usually unaffected. Males who inherit the pathogenic variant are affected.

\section{Limits of the test}

The test is limited by current scientific knowledge regarding the gene and disease.

Analytical sensitivity (proportion of positive tests when the genotype is truly present) and specificity (proportion of negative tests when the genotype is not present)

NGS: Analytical sensitivity $>99.99 \%$, with a minimum coverage of 10X; Analytical specificity $99.99 \%$.

SANGER: Analytical sensitivity >99.99\%; Analytical specificity 99.99\%.

MLPA: Analytical sensitivity $>99.99 \%$; Analytical specificity $99.99 \%$.

Clinical sensitivity (proportion of positive tests if the disease is present) and clinical specificity (proportion of negative tests if the disease is not present)

Clinical sensitivity: $20-25 \%$ for familial non syndromic AAT $[18,19]$. In syndromic forms, clinical sensitivity depends on the specific clinical criteria.

Clinical specificity is estimated at approximately $99 \%(19,20)$.

\section{Prescription appropriateness}

The genetic test is appropriate when:

a) the patient meets the diagnostic criteria for Large-caliber vessel aneurysms;

b) the sensitivity of the test is greater than or equal to that of tests described in the literature.

\section{Clinical utility}

Clinical management Utility

Confirmation of clinical diagnosis Yes

Differential diagnosis Yes

Couple risk assessment Yes

Availability of clinical trials can be checked on-line at https://clinicaltrials.gov/

\section{References}

1. Gusani R, Shukla R, Kothari S, Bhatt R, Patel J. Inferior vena cava aneurysm presenting as deep vein thrombosis - A case report. International Journal of Surgery Case Reports 2016; 29: 123-25.

2. Erkanli K, Yazici P, Bakir I. Pulmonary vein aneurysm secondary to mitral regurgitation: rare and confusing lesion. Thorac Cardiovasc Surg 2014; 62(1): 83-4. 
3. De Paepe A, Devereux RB, Dietz HC, Hennekam RC, Pyeritz RE. Revised diagnostic criteria for the Marfan syndrome. Am J Med Genet 1996; 62(4): 417-26.

4. Gillis E, Van Laer L, Loeys BL. Genetics of thoracic aortic aneurysm: at the crossroad of transforming growth factor- $\beta$ signaling and vascular smooth muscle cell contractility. Circ Res 2013; 113(3): 327-40.

5. Lindsay ME, Dietz. Lessons on the pathogenesis of aneurysm from heritable conditions. Nature 2011; 473(7347): 308-16.

6. Ramanath VS, Oh JK, Sundt TM 3rd, Eagle KA . Acute aortic syndromes and thoracic aortic aneurysm. Mayo Clin Proc 2009; 84(5): 465-81.

7. Blanchard JF, Armenian HK, Friesen PP. Risk factors for abdominal aortic aneurysm: results of a case-control study. Am J Epidemiol 2000; 151(6): 575-83.

8. Biddinger A, Rocklin M, Coselli J, Milewicz DM. Familial thoracic aortic dilatations and dissections: a case control study. J Vasc Surg 1997; 25(3): 506-11.

9. Albornoz G, Coady MA, Roberts M, Davies RR, Tranquilli M, Rizzo $J A$, Elefteriades JA. Familial thoracic aortic aneurysms and dissections--incidence, modes of inheritance, and phenotypic patterns. Ann Thorac Surg 2006; 82(4): 1400-05.

10. Singh K, Bønaa KH, Jacobsen BK, Bjørk L, Solberg S. Prevalence of and risk factors for abdominal aortic aneurysms in a population-based study: The Tromsø Study. Am J Epidemiol 2001; 154(3): 236-44.

11. Thompson RW, Parks WC. Role of matrix metalloproteinases in abdominal aortic aneurysms. Ann N Y Acad Sci 1996; 800: 157-74.

12. Sandford RM, Bown MJ, London NJ, Sayers RD. The genetic basis of abdominal aortic aneurysms: a review. Eur J Vasc Endovasc Surg 2007; 33(4): 381-90.

13. Rahimi S. Abdominal Aortic Aneurysm Clinical Presentation. Updated: 1994-2017 by WebMD LLC. available from: https://emedicine.medscape.com/article/1979501-clinical.

14. Doyle AJ, Doyle JJ, Bessling SL, Maragh S, Lindsay ME, Schepers D, Gillis E, Mortier G, Homfray T, Sauls K, Norris RA, Huso ND, Leahy D, Mohr DW,Caulfield MJ, Scott AF, Destrée A, Hennekam RC, Arn
PH, Curry CJ, Van Laer L, McCallion AS, Loeys BL, Dietz HC. Mutations in the TGF- $\beta$ repressor SKI cause Shprintzen-Goldberg syndrome with aortic aneurysm. Nat Genet 2012; 44(11): 1249-54

15. Guo, D, Gong, L, Regalado, ES, Santos-Cortez, RL, Zhao, R, Cai B, Veeraraghavan S, Prakash SK, Johnson RJ, Muilenburg A, Willing M,Jondeau G, Boileau C, Pannu H, Moran R, Debacker J; GenTAC Investigators, National Heart, Lung, and Blood Institute Go Exome Sequencing Project; Montalcino Aortic Consortium, Bamshad MJ, Shendure J, Nickerson DA, Leal SM, Raman CS, Swindell EC, Milewicz DM. MAT2A mutations predispose individuals to thoracic aortic aneurysms. Am. J. Hum. Genet 2015; 96(1): 170-77.

16. Micha D, Guo DC, Hilhorst-Hofstee Y, van Kooten F, Atmaja D, Overwater E, Cayami FK, Regalado ES, van Uffelen R, Venselaar $\mathrm{H}$, Faradz SM, Vriend G, Weiss MM, Sistermans EA, Maugeri A, Milewicz DM, Pals G, van Dijk FS. SMAD2 Mutations Are Associated with Arterial Aneurysms and Dissections. Hum Mutat 2015; 36(12): 1145-49.

17. Ziganshin BA, Bailey AE, Coons C, Dykas D, Charilaou P, Tanriverdi LH, Liu L, Tranquilli M, Bale AE, Elefteriades JA. Routine Genetic Testing for ThoracicAortic Aneurysm and Dissection in a Clinical Setting. Ann Thorac Surg 2015; 100(5): 1604-11.

18. Milewicz DM, Regalado E. Heritable Thoracic Aortic Disease Overview. [Updated 2016 Dec 29]. In: Adam MP, Ardinger HH, Pagon RA, et al., editors. GeneReviews ${ }^{\circledR}$ [Internet]. 1993-2017. Available from: https://www.ncbi.nlm.nih.gov/books/NBK1120/.

19. Arslan-Kirchner M, Arbustini E, Boileau $C$, Charron $\mathrm{P}$, Child $\mathrm{AH}$, Gwenaelle Collod-Beroud, Julie De Backer, Anne De Paepe, Anna Dierking, Laurence Faivre, Sabine Hoffjan, Guillaume Jondeau, Britta Keyser, Bart Loeys, Karin Mayer, Peter N Robinson, and Jörg Schmidtke. Clinical utility gene card for: Hereditary thoracic aortic aneurysm and dissection including next-generation sequencing-based approaches. Eur J Hum Genet 2016; 24(1): 146-150.

20. Chen B, Gagnon M, Shahangian S, Anderson NL, Howerton DA, Boone JD; Centers for Disease Control and Prevention (CDC). Good Laboratory Practices for Molecular Genetic Testing for Heritable Diseases and Conditions. MMWR Recomm Rep 2009; 58(RR-6): 1-37. 